\title{
Reflexos da cooperação no desempenho de franquias: mediação do compartilhamento de informações da confiança interorganizacional
}

\section{Reflexes of cooperation in the performance of franchises: mediation of information sharing and interorganizational trust}

\author{
Rosiele Bescorovaine \\ Universidade Federal do Paraná (UFPR) \\ email: rosiele_besc@hotmail.com
}

\section{Ilse Maria Beuren}

Universidade Federal de Santa Catarina (UFSC)

email:ilse.beuren@gmail.com

\section{RESUMO}

Este estudo analisa os reflexos da cooperação no desempenho de franquias, mediados pelo compartilhamento de informações e pela confiança interorganizacional. Uma survey foi realizada com gestores de empresas franqueadas do setor de comércio varejista de produtos farmacêuticos e se obteve 151 respostas válidas. Para análise dos dados utilizou-se a modelagem de equações estruturais. Os resultados revelaram que, na percepção dos gestores, os construtos da Teoria da Cooperação estão presentes nas interações interfirmas com a franqueadora, exceto a disposição para adaptação à mudanças. Destacam-se o compartilhamento de informações e a confiança interorganizacional, por estimularem comportamentos cooperativos para melhorar o desempenho organizacional. Conclui-se que o compartilhamento de informações e a confiança interorganizacional, quando mediadoras do efeito da cooperação, refletem no desempenho financeiro e não financeiro das franquias. Além dos esforços coordenados de cooperação, elucida-se aos franqueados maneiras de reforçar seu relacionamento interfirmas, pelo compartilhamento de informações e pela confiança interorganizacional, com reflexos no desempenho das franquias.

Palavras-Chave: Cooperação interfirmas; Compartilhamento de informações; Confiança inteorganizacional; Desempenho organizacional.

\section{ABSTRACT}

This study analyzes the reflexes of cooperation in the performance of franchises, mediated by information sharing and interorganizational trust. A survey was conducted with managers of franchisees in the retail sector of pharmaceutical products, obtaining 151 valid answers. For data analysis structural equation modeling was used. The results revealed that, according to the managers' perception, the constructs of the Cooperative Theory are present in the interfirm interactions with the franchisor, except for the willingness to adapt to changes. Information sharing and interorganizational trust stand out as they stimulate cooperative behavior to improve the organizational performance. It is concluded that information sharing and interorganizational trust, when mediating the effect of cooperation, reflect into financial and non-financial performance of franchises. In addition to the coordinated cooperation efforts, franchisees are encouraged to strengthen their interfirm relationship by sharing information and interorganizational trust, reflecting on the performance of the franchises.

Key-words: Interfirm cooperation; Information sharing; Interorganizational trust; Organizational performance. 


\section{INTRODUÇÃO}

A adesão a modelos de cooperação pode ser interessante, especialmente em cenários de empresas assoladas pelas dificuldades de assegurar sua competitividade e/ou que exprimem uma destoante realidade (BALESTRIN; VERCHOORE; REYES JR, 2010; WEBER; HEIDENREICH, 2018). As relações de cooperação interorganizacional objetivam alavancar os negócios das empresas (DEKKER; DING; GROOT, 2016). Podem ser definidas como ações similares ou complementares coordenadas, tomadas por firmas nas relações interdependentes, para o alcance de resultados mútuos e individuais, com a expectativa de reciprocidade de longo prazo (ANDERSON; NARUS, 1990).

Comportamentos cooperativos são incentivados para auferir um nível de vantagem competitiva que individualmente não seria possível (BRITO; BRITO; HASHIBA, 2014). Para isso, empresas que possuem alguns, mas nem todos os recursos necessários, combinam recursos com outra organização, utilizando um acordo de cooperação (COMBS; KETCHEN, 1999). A cooperação interorganizacional entre empresas formalmente independentes, que se relacionam, tende a mostrar efeitos positivos, pois é capaz de formar competências, redesenhar estratégias e explorar recursos complementares (MOURITSEN; THRANE, 2006). Esses benefícios melhoram o desempenho (MAHAMA, 2006) das empresas como um todo.

No entanto, evidências empíricas sugerem que os gestores tendem a ponderar medidas financeiras mais fortemente do que medidas não financeiras (CARDINAELS; VEEN-DIRKS, 2010). Medidas financeiras (ex.: crescimento das vendas, lucratividade) partilham de uma orientação comum que tira o enfoque de seus formadores (ex.: comprometimento dos fornecedores, experiência no ramo, competências do capital intelectual). Anthony, Govindarajan e Dearden (1998) argumentam que os gestores estão mais familiarizados com pressões financeiras, o que reforça a tendência de confiar mais em indicadores financeiros (ITTNER; LARCKER, 1998). Com o propósito de neutralizar essa percepção, Kaplan e Norton (1992) propuseram o uso de métricas financeiras e não financeiras de desempenho, ampliando, assim, a base para a tomada de decisão.
Para além do desempenho financeiro e não financeiro, a confiança é um recurso chave sob a ótica da coordenação das expectativas e interações entre os atores econômicos em cooperação (BACHMANN, 2001; HERZ et al., 2016). A relevância da confiança interorganizacional está diretamente relacionada ao surgimento de formas híbridas de coordenação, sendo que essas estruturas trabalham como entidades econômicas independentes, cada uma especializada em habilidades específicas, as quais cooperam entre si por meio do intercâmbio de informações (CHEN; LIN; YEN, 2014).

O compartilhamento de informações na cooperação pode favorecer a criação de conhecimentos estratégicos voltados à competitividade das organizações (BALESTRIN; VERCHOORE; REYES JR, 2010; PARTANEN et al., 2020). Para Atallah (2003), a partilha de informações contribui para a cooperação, mas de forma involuntária pode ocorrer vazamento de informações a terceiros. Na pesquisa realizada em ambientes interorganizacionais o autor encontrou relações positivas entre compartilhamento de informações, desempenho e redução de custos.

Mahama (2006) também constatou em sua pesquisa que as dimensões da cooperação (resolução conjunta de problemas, uso do poder, disposição para adaptação à mudanças e compartilhamento de informações) exercem influência positiva e significativa no desempenho organizacional. O estudo realizado por Fisher, Frederickson e Peffer (2002), sobre compartilhamento de informações para a resolução conjunta de problemas e uso do poder, aponta que a partilha de informações entre os indivíduos reduz a probabilidade do poder ser exercido e facilita a resolução conjunta de problemas.

As dimensões da Teoria da Cooperação, testadas inicialmente no estudo de Heide e Miner (1992) e depois corroboradas por Mahama (2006), presume-se que perdurem. Porém, novas interações entre essas variáveis (resolução conjunta de problemas, uso do poder, disposição para adaptação à mudanças e compartilhamento de informações) foram sugeridas e testadas. Neste estudo, o compartilhamento de informações foi considerado um construto separado devido ao seu poder de explicação estatístico em estudos empíricos (MAHAMA, 2006; BEUREN; DAL VESCO, 2021), sua importância frente a outras 
dimensões da cooperação dado a necessidade de comunicação entre as firmas (MOHR; SPEKMAN, 1994) e de criar mecanismos eficientes para a resolução de conflitos (HEIDE; MINER, 1992).

Nesta perspectiva, a pergunta que norteia esta pesquisa é: Quais os reflexos da cooperação no desempenho de franquias, mediados pelo compartilhamento de informações e pela confiança interorganizacional? Diversas são as formas de relação interorganizacional, mas uma que importa para este estudo é o sistema franchising. Em sistemas franchising duas unidades de negócio podem integrar uma mesma rede, ainda que seus pontos de venda sejam próximos e possuam a mesma clientela. Nesse caso, ambas assumem comportamentos cooperativos em seu relacionamento com o franqueador, e de concorrência de um franqueado com o outro, devido sua localização geográfica. Portanto, as relações interfirmas podem ser cooperativas e competitivas (TIDSTROM; HAGBERG-ANDERSSON, 2012).

O estudo apresenta contribuição teórica ao abranger elementos de um vasto campo de pesquisas de relações interfirmas em aberto. Tal envolve na perspectiva do franqueado o compartilhamento de informações entre as partes envolvidas na cooperação (MAHAMA, 2006) e a confiança interorganizacional (RING; VAN DE VEN, 1994), ambas como mediadoras do efeito da cooperação no desempenho (ASHNAI et al., 2016). Do ponto de vista da prática gerencial, esta pesquisa contribui para o debate sobre as relações interfirmas, em particular do sistema franchising, trazendo à tona a complexidade das rotinas interorganizacionais e o deslinde de componentes que podem reforçar a cooperação e refletir no desempenho organizacional financeiro e não financeiro.

\section{REFERENCIAL TEÓRICO E HIPÓTESES}

A Teoria da Cooperação pressupõe que pessoas ou grupos agem em conjunto de forma coordenada para perseguir objetivos comuns (BROWNING; BEYER; SHETLER, 1995). A cooperação interfirmas, foco deste estudo, envolve a realização de atividades coordenadas, similares ou complementares, desempenhadas por empresas em relacionamentos inter- dependentes, com a finalidade de obter resultados mútuos ou unilaterais (ANDERSON; NARUS, 1990). Segundo Balestrin, Verchoore e Reyes Jr (2010), a cooperação visa reunir ações que permitam às organizações envolvidas melhor adequação ao ambiente competitivo. A cooperação interorganizacional ocorre quando as partes envolvidas optam por agir em conjunto em prol de um objetivo maior, unindo suas competências, de forma a ampliar a eficiência organizacional do conjunto (ABRADE, 2010).

Uma das motivações ao comportamento cooperativo está na melhora percebida da eficiência econômica das organizações participantes (HEIDE; JONH, 1990; DEKKER; DING; GROOT, 2016). Também para minimizar as incertezas face à escassez de recursos, e maximizar a reciprocidade entre as partes envolvidas nas transações existentes (AXELROD, 1984; PARTANEN et al., 2020). O insucesso de alianças interorganizacionais pode decorrer da falta de comportamentos cooperativos entre os participantes (SMITH; CARROL; ASHFORD, 1995; WEBER; HEIDENREICH, 2018). De modo geral, três abordagens distintas são utilizadas para a concepção e definição de cooperação: psicológica, comportamental e dimensional (CHEN; CHEN; MEINDL, 1998).

A abordagem psicológica decorre de motivos psicológicos dos participantes, e foi preconizada por Mead (1932). Nessa dimensão, a cooperação consiste no ato de trabalhar conjuntamente para um objetivo estabelecido, assim o indivíduo inserido no grupo tende a ter comportamentos coerentes e engajados na atividade coletiva. Desse modo, os conceitos de cooperação e competição representam diferentes motivações psicológicas.

A abordagem comportamental concentra-se nas relações sociais em contextos organizacionais. Essa abordagem foi proposta por Deutsch (1949) e posteriormente desenvolvida por Tjosvold (1988), que amplia o preconizado por Mead (1932), pois altera o foco na concepção do contexto cultural, para concentrar-se na natureza das relações existentes entre os participantes. Segundo Barnard (1938), ela fornece um conceito amplo do comportamento cooperativo, pois os processos de gestão são conduzidos por ações cooperativas dos participantes, para facilitar e manter a cooperação interorganizacional, assim as ações são individuais, mas os propósitos coletivos. Axelrod 
(1984) destaca que, além de finalidades em conjunto, os indivíduos devem perceber algum retorno em seu desempenho.

A abordagem dimensional apresenta quatro formas de interação: (i) compartilhamento de informações, ideias e outros recursos; (ii) assistência; (iii) resolução de problemas e conflitos de forma coletiva; e (iv) apoio e incentivo aos outros (TJOSVOLD, 1988). Nesta abordagem, a presença de comportamentos cooperativos é ditada pela dinâmica das interações coletivas, e não pela existência de uma atividade coletiva (CHEN; CHEN; MEINDL, 1998). Assim, o êxito de comportamentos cooperativos exige: coordenação, comunicação e divisão clara das atividades a serem desenvolvidas.

Não há consenso na literatura quanto às dimensões de análise da cooperação, contudo, as proposições amplamente difundidas e convergentes são as de Heide e Miner (1992), corroboradas por Mahama (2006), a saber: flexibilidade; compartilhamento de informações; resolução conjunta de problemas; e restrição ao uso do poder. Esses quatro elementos indicam que os atores (indivíduos, grupos ou organizações) comprometem-se a canalizar esforços de interação e comprometimento no intuito de benefício mutuo. A Teoria da Cooperação anseia explicar tais relacionamentos, porém admite que uma de suas dimensões (compartilhamento de informações) possa não ser apenas um formativo da teoria, mas sim um condutor entre a cooperação e o desempenho (HEIDE; MINER, 1992), o que sugere que essa dimensão pode resultar em um nexo de causalidade entre as outras dimensões.

\subsection{Cooperação Interfirmas e Desempenho Organizacional}

Estudos pregressos (MAHAMA, 2006; DEKKER; DING; GROOT, 2016; BEUREN; DAL VESCO, 2021) revelam que nem todos os comportamentos cooperativos têm impactos semelhantes e positivos no desempenho. Apesar do apoio empírico para a existência de relações positivas entre cooperação e desempenho, não há consenso quanto aos resultados encontrados, como pode ser observado nas pesquisas que seguem.
Turnbull, Oliver e Wilkinson (1992) apontaram as dificuldades enfrentadas por empresas automobilísticas do Reino Unido em replicar práticas cooperativas presentes em empresas japonesas e, ainda, que os comportamentos cooperativos são dependentes do contexto em que estão inseridos, pois elementos como a estrutura da organização, o mercado competitivo e a cultura podem ser fatores moderadores entre cooperação e desempenho.

Pesquisa realizada por Burnes e New (1997) no Reino Unido em um grupo industrial automobilístico, porém com uma perspectiva mais crítica, adverte quanto aos demasiados efeitos benéficos apontados para relações de cooperação. Combs e Ketchen (1999) examinaram as implicações da cooperação no desempenho de 94 redes de restaurantes de capital aberto, e constataram que o efeito da cooperação sobre o desempenho é dependente do contexto relacional das empresas.

Vereecke e Muylle (2006) também investigaram acerca da relação entre cooperação e desempenho, e identificaram apenas uma relação fraca. Pesquisaram 730 empresas espanholas utilizando a Teoria do Capital Social, e constataram que a cooperação tem efeito positivo no desempenho até certo ponto, e que o excesso percebido de comportamentos cooperativos passa a apresentar efeito negativo sobre o desempenho.

Mahama (2006) corrobora que a cooperação tem impacto positivo no desempenho e infere que o incentivo para cooperar ocorre quando os participantes percebem melhora no seu desempenho individual em comparação ao possível resultado obtido caso não houvesse ajuda mútua. Palmatier, Dant e Grewal (2007) coadunam acerca da cooperação influenciar positivamente no desempenho e que as relações interorganizacionais integram construções focais que propõem melhor desempenho a partir de relacionamentos que investem em tempo, recursos financeiros, conhecimentos e capacidades.

Brito, Brito e Hashiba (2014) analisaram os efeitos das dimensões para cooperação propostas por Heide e Miner (1992) - flexibilidade, compartilhamento de informações, resolução conjunta de problemas e restrição ao uso do 
poder - no desempenho organizacional, tendo como amostra 124 fabricantes de embalagens. A flexibilidade não apresentou efeito significativo, a resolução conjunta de problemas apresentou efeito negativo, enquanto o compartilhamento de informações e a restrição ao uso do poder apresentaram efeitos positivos e significativos no desempenho.

Os estudos apresentados focaram ganhos financeiros, porém, Heide e Miner (1992) também preveem ganhos não financeiros. Uma análise puramente quantitativa das interações pode limitar-se a considerar as informações fornecidas por apenas uma das partes (CARDINAELS; VEEN-DIRKS, 2010). Todavia, em relacionamentos interorganizacionais dois importantes fatores estão presentes nas interações objetivando a melhora do desempenho financeiro e não financeiro: comprometimento entre as partes e mutua confiança (BEUGELSDIJK; KOEN; NOORDERHAVEN, 2009).

Diversas podem ser as medidas financeiras (ex.: expansão do negócio, novos clientes, competitividade, resultado econômico-financeiro) para aferir o sucesso da cooperação (BURNES; NEW, 1997; COMB; KETCHEN, 1999; VEREECKE; MUYLLE, 2006; PALMATIER; DANT; GREWAL, 2007). Por sua vez, as medidas não financeiras podem advir da percepção dos fraqueados, no caso de sistemas franchising, acerca de ganhos/ perdas (ex.: aprendizagem, contatos valiosos, percepção das necessidades dos clientes antes dos concorrentes) do seu negócio a partir dos esforços coordenados com o franqueador (SELNES, SALLIS, 2003; BEUGELSDIJK; KOEN; NOORDERHAVEN, 2009; ASHNAI et al., 2016). Assim, com base nos argumentos teórico-empíricos apresentados formularam-se as seguintes hipóteses:

H1a: Há uma relação positiva entre a cooperação em franquia e o desempenho financeiro do franqueado.

H1b: Há uma relação positiva entre a cooperação em franquia e o desempenho não financeiro do franqueado.

\subsection{Cooperação Interfirmas e Compartilhamento de Informações}

O compartilhamento de informações trata da disposição dos participantes proporcionar troca de informações úteis em um ambiente de cooperação interfirmas (TJOSVOLD, 1988; PARTANEN et al., 2020), e refere-se à medida que a informação é transmitida aos pares em um relacionamento interorganizacional (MOHR; SPEKMAN, 1994; CANNON; PERREAULT, 1999; MAHAMA, 2006). O grau de compartilhamento de informações pode variar conforme o tipo de relacionamento interfirmas (HEIDE; MINER, 1992), por exemplo, no caso de produtos altamente personalizados, a produção requerer um maior fluxo de partilha de informações para cumprir os padrões estabelecidos.

Nesta perspectiva, Combs (1993) propõe um framework de compartilhamento de informações, um modelo voltado a um ambiente de pesquisa e desenvolvimento, com o qual as empresas podem aumentar a probabilidade de descobrir um novo produto pelo compartilhamento de informações. Cálculos são propostos para demonstrar que a cooperação tende a aumentar a possibilidade de melhorar o desempenho, na premissa de que a comunicação interfirmas resulta do esforço coordenado pelas partes envolvidas para criar mecanismos de resolução conjunta de problemas.

Chen, Lin e Yen (2014) veem a partilha de conhecimento interorganizacional como um fator crucial para a coordenação colaborativa de recursos, alocação e integração entre os membros de uma cadeia de fornecimento. Destacam ainda que o compartilhamento de informações é positivo para as organizações, por proporcionar a expansão do rol de recursos à disposição, passar a oferecer produtos de valor agregado, detectar oportunidades emergentes e capturar os benefícios de negócios num ambiente de mercado competitivo.

Essas interações, desde que claramente definidas, tendem a ser facilitadoras para a resolução de problemas entre os atores (HEIDE; MINER, 1992; WEBER; HEIDENREICH, 2018). No entanto, a partilha pode também exercer efeito negativo sobre a cooperação, pois em uma relação assimétrica a dependência das partes pode não ser recíproca, de modo que uma das 
partes exerça poder sobre a outra, mas não vice-versa (HEIDE; MINER, 1992; WEBER; HEIDENREICH, 2018). A partir do exposto, formulou-se a segunda hipótese:

H2: Há uma relação positiva entre a cooperação em franquia e o compartilhamento de informações.

\subsection{Cooperação e Confiança Interorganizacional}

Mayer, Davis e Schoorman (1995, p. 712) definem confiança como a "vontade de uma das partes ser vulnerável às ações de outra parte com base na expectativa de que o outro irá executar determinada ação importante para o cedente, independentemente da capacidade de monitorar ou controlar essa outra parte". A confiança é um estado psicológico que compreende a intenção de aceitar a vulnerabilidade baseada em expectativas positivas das intenções ou comportamentos de outra (ROUSSEAU et al., 1998).

Confiança é um fenômeno interpessoal (GULATI, 1995). Ao conceber as organizações como aglomerações de pessoas é possível considerar de forma análoga a existência de confiança entre empresas nas transações econômicas (ZUCKER, 1986). A delimitação de confiança é complexa, uma vez que se trata de um conceito meso, que associa processos de níveis psicológicos e dinâmicas de grupo com arranjos de níveis institucionais (HOUSE; ROUSSEAU; THOMAS-HUNT, 1995). No contexto interorganizacional, a confiança refere-se ao grau que os membros da organização confiam nas empresas parceiras (GULATI, 1995). A confiança é um catalisador que facilita interações de negócios estratégicos e partilha de conhecimentos entre as empresas (CHEN; LIN; YEN, 2014).

A confiança em empresas parceiras (RING; VAN DE VEN, 1994) e a crença de que seus parceiros irão realizar suas atividades da melhor maneira, beneficia a relação de troca (SMITH; CARROL; ASHFORD, 1995) e fortalece a capacidade de foco da empresa para prever o comportamento de seus parceiros. Para House,
Rousseau e Thomas-Hunt (1995), a confiança interorganizacional é a crença subjetiva com que as partes da organização avaliam coletivamente se a população de organizações irá realizar transações potenciais confiantes conforme suas expectativas, independente da sua capacidade de monitorá-los totalmente.

Nas últimas décadas, a confiança interorganizacional tem recebido atenção nas pesquisas pelo fato dela contribuir para o comportamento cooperativo (GAMBETTA, 1988; HERZ et al., 2016), promover formas de organização adaptativas, como relações de rede (MILES; SNOW, 1992; WEBER; HEIDENREICH, 2018), reduzir conflitos prejudiciais, diminuir custos de transação, facilitar interação de grupos de trabalho e promover respostas eficazes em ambientes de incerteza (BALESTRIN; VERCHOORE; REYES JR, 2010). A confiança em transações comerciais não é unicamente de natureza econômica e influencia as transações entre empresas (ANDERSON; THOMSPON, 2004; PARTANEN et al., 2020).

A confiança interorganizacional pode ser mais relevante do que aspectos legais (contratos formais e acordos) da relação (MCAULAY, 1963). Segundo Fukuyama (1996), quanto maior o grau de confiança nas organizações, maior a cooperação espontânea e menor a necessidade de aparatos legais, como sistemas de normas e regulamentos, negociados ou coercitivos, para garantir a cooperação. Confiança interorganizacional é uma pré-condição para a troca relacional (CHEN; LIN; YEN, 2014), porém, é singularmente relevante em situações ambíguas, comumente encontradas nas relações interfirmas. Nesse aspecto, a literatura sugere algumas métricas (ex.: competência do parceiro, correspondência às expectativas, cumprimento de promessas, relação honesta e verdadeira, suporte necessário) para aferir a confiança interorganizacional (RING; VAN DE VEN, 1994; ROUSSEAU et al., 1998; CHEN; LIN; YEN, 2014). Diante do exposto, formulou-se a terceira hipótese:

H3: Há uma relação positiva entre a cooperação em franquia e a confiança interorganizacional. 


\subsection{Cooperação e Desempenho: Efeitos do Compartilhamento de Informações e da Confiança Interorganizacional}

Empresas se envolvem em relações interorganizacionais na expectativa de potenciais ganhos provindos de ações conjuntas (BRITO; BRITO; HASHIBA, 2014). Pesquisas apontam que há relação significativa com desempenho, quando associado com: (i) partilha de informações (CHEN; CHEN; MEINDL, 1998; PARTANEN et al., 2020); (ii) difusão do conhecimento (MOURITSEN; THRANE, 2006); (iii) adaptabilidade à mudanças (HEIDE; MINER, 1992; MAHAMA, 2006); (iv) restrição ao uso do poder (HEIDE; MINER, 1992); e (v) capacidade de exercer o poder (MAHAMA, 2006).

Estudos anteriores sugerem que maior partilha de informações reduz a incerteza e melhora o nível de confiança e compromisso no relacionamento interfirmas (ANDERSON; WEITZ, 1992; KWON; SUH, 2004; HERZ et al., 2016). O compartilhamento de informações apresenta influência significativa na resolução de problemas (TJOSVOLD, 1988; HEIDE; MINER, 1992; CANNON; PERREAULT, 1999; CHEN; LIN; YEN, 2014), disposição para adaptação à mudanças (HEIDE; MINER, 1992; MAHAMA, 2006) e restrição ao uso do poder (HEIDE; MINER, 1992; MAHAMA, 2006).

Heide e John (1990) previam que o compartilhamento de informações interorganizacionais exerce efeito sobre o desempenho. Essas trocas ocorrem de forma ordenada, e com ônus menor do que individualmente. $\mathrm{O}$ estudo sugere que a melhora do desempenho ocorre quando os indivíduos o percebem e, com isso, mantêm comportamentos cooperativos. Dekker, Ding e Groot (2016), ao analisar como os objetivos colaborativos das empresas influenciam o uso das práticas de gestão de desempenho nas relações interfirmas, encontraram que o compartilhamento de informações é usado como prática complementar na gestão de desempenho de relacionamentos interorganizacionais.

Com base nestes estudos é que se presume que o compartilhamento de informações pode resultar em nexo de causalidade entre as outras dimensões da Teoria da Cooperação. Assim, formularam-se as seguintes hipóteses:
H4a: Há uma relação positiva entre a cooperação em franquia e o desempenho financeiro do franqueado, mediada pelo compartilhamento de informações.

H4b: Há uma relação positiva entre a cooperação em franquia e o desempenho não financeiro do franqueado, mediada pelo compartilhamento de informações.

A confiança também pode ser um importante preditor de partilha de conhecimentos, visto que afeta a capacidade percebida, a benevolência, a integridade e a previsibilidade dos parceiros comerciais para cumprir as normas do negócio (MCKNIGHT; CHOUDHURY; KACMAR, 2002). A confiança reflete no escopo e profundidade das relações entre parceiros, o que pode fortalecer a parceria e melhorar a partilha de conhecimentos (HERZ et al., 2016).

Segundo Morgan e Hunt (1994), o compromisso e a confiança são construções focais que viabilizam a compreensão da cooperação e do desempenho interorganizacional, sendo que o compromisso é precursor fundamental para melhorar o desempenho financeiro. Testes realizados, isolados ou conjuntamente, de interações entre o compromisso, confiança e desempenho financeiro, demonstraram que ambos exercem efeitos positivos sobre o desempenho financeiro e não financeiro. Assim, formularam-se as hipóteses que seguem:

H5a: Há uma relação positiva entre a cooperação em franquia e o desempenho financeiro do franqueado, mediada pela confiança interorganizacional.

H5b: Há uma relação positiva entre a cooperação em franquia e o desempenho não financeiro do franqueado, mediada pela confiança interorganizacional.

Na Figura 1 apresenta-se o modelo teórico da pesquisa, onde são destacados os construtos e as hipóteses da pesquisa.

Observa-se na Figura 1, a relação indireta entre a cooperação (resolução conjunta de problemas, uso do poder e disposição para adaptação à mudanças), como variável antecedente e independente; a confiança interorganizacional e o compartilhamento de informações como variáveis mediadoras; e o desempenho organizacional como variável dependente. 


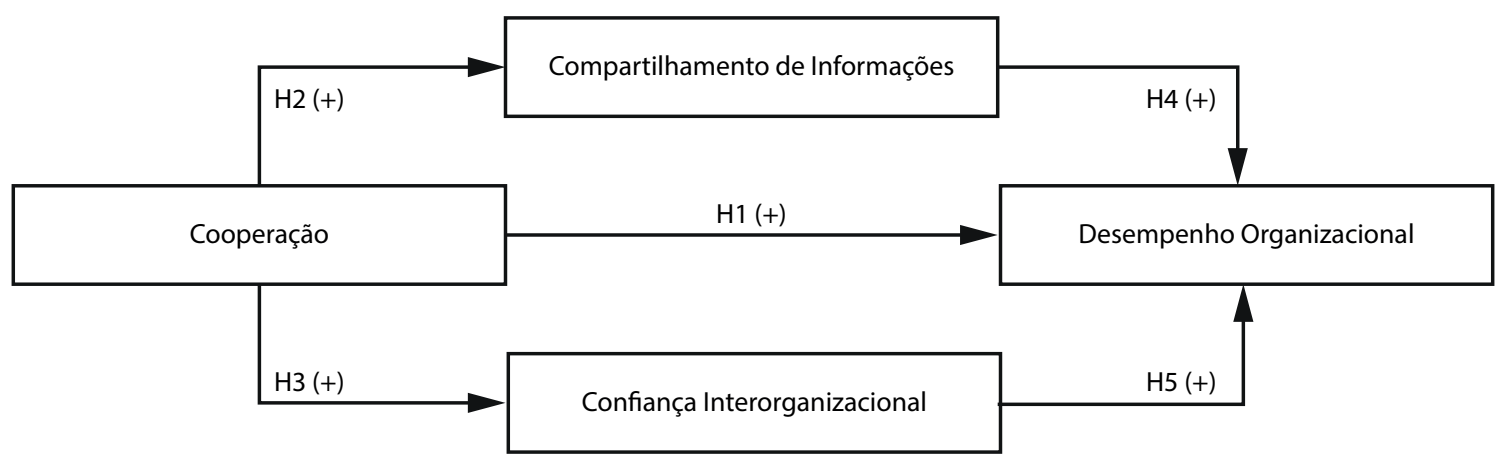

Figura 1 Modelo teórico da pesquisa

Fonte: Elaboração própria.

\section{PROCEDIMENTOS METODOLÓGICOS}

Uma pesquisa de levantamento foi realizada com gestores de empresas franqueadas do ramo farmacêutico do segmento varejista, filiadas ao Sindicato do Comércio Varejista de Produtos Farmacêuticos do Estado do Paraná. Trata-se de um setor assolado pelas dificuldades inerentes à competitividade e exprime uma realidade ostensiva frente às grandes organizações e, de forma a suprir essas lacunas, empresas unem-se em sistemas franchising. Esses aspectos se coadunam com o campo alvo desta pesquisa, sendo que o recorte foi realizado pela acessibilidade para a coleta de dados por meio de questionário eletrônico.

Os dados dos franqueados foram fornecidos pelo Sindicato mediante compromisso de preservação do sigilo dos envolvidos. A compilação dos dados revelou uma população de 506 franqueados, detentores de contrato com uma franqueadora. Optou-se pela exclusão das empresas sem atividade, ou seja, franqueados em fase de implementação das instalações físicas ou em processo de baixa ou mudança.

Ao estimar a percentagem populacional a $50 \%$ de significância, usando-se uma margem de erro de 5\%, a amostra mínima foi de 81 respondentes. Para conseguir esse número de respostas, contatou-se o Sindicato para expor a natureza e os objetivos da pesquisa, além da solicitação para o envio do questionário ao e-mail das unidades franqueadas associadas. Dos 160 questionários respondidos, 151 foram válidos, perfazendo $30 \%$ da população.
Os construtos da pesquisa e suas respectivas variáveis são apresentados no Quadro 1.

Os construtos sobre relações interfirmas consistem de elementos chaves da Teoria da Cooperação, enquanto o desempenho organizacional como um consequente. A partir dos construtos da pesquisa e das respectivas variáveis foi estruturado o instrumento de pesquisa, composto de 32 questões, em escala tipo Likert de sete pontos ( 1 = discordo totalmente a 7 = concordo totalmente), em consonância com estudos que fundamentam o referencial teórico.

Com vistas na validação externa, o questionário foi submetido a dois pré-testes para eliminar possíveis inconsistências e minimizar dificuldades de compreensão. Para a realização do primeiro pré-teste, o questionário foi aplicado a dois pesquisadores da área e, a partir das suas sugestões, fizeram-se alterações. O segundo pré-teste foi realizado com três profissionais da área, com perfil semelhante ao da amostra desta pesquisa, com intuito de avaliar os termos técnicos empregados no questionário, para que fossem compreensíveis.

Para a coleta de dados, o questionário foi disponibilizado no formato on-line na plataforma Google Docs. O instrumento ficou disponível para recebimento de respostas no período de janeiro a fevereiro de 2016. O envio do questionário aos franqueados foi realizado pelo departamento de comunicação do Sindicato, que encaminhou para sua lista de contatos um e-mail com um texto de apresentação da pesquisa e o convite para participação da survey, além de informar o link para acesso do questionário. 
Quadro 1 Construtos de pesquisa

\begin{tabular}{l|l|l}
\hline \multicolumn{1}{c|}{ Construtos } & \multicolumn{1}{c|}{ Variáveis } & \multicolumn{1}{c}{ Referências } \\
\hline \multirow{2}{*}{ Cooperação } & $\begin{array}{l}\text { Resolução conjunta de problemas } \\
\text { (RESCP) }\end{array}$ & $\begin{array}{l}\text { Heide e Miner (1992); } \\
\text { Mahama (2006). }\end{array}$ \\
\cline { 2 - 3 } & Uso do poder (USOP) & $\begin{array}{l}\text { Heide e Miner (1992); } \\
\text { Mahama (2006). }\end{array}$ \\
\cline { 2 - 3 } & $\begin{array}{l}\text { Disposição para adaptação à mudanças } \\
\text { (DMUD) }\end{array}$ & $\begin{array}{l}\text { Heide e Miner (1992); } \\
\text { Mahama (2006). }\end{array}$ \\
\hline \multirow{2}{*}{ Compartilhamento de Informações } & Compartilhamento de informações (COMP) & $\begin{array}{l}\text { Tjosvold (1988); } \\
\text { Heide e Miner (1992); } \\
\text { Cannon e Perreault (1999); } \\
\text { Mahama (2006). }\end{array}$ \\
\hline \multirow{2}{*}{ Confiança Interorganizacional } & Confiança interorganizacional (CONF) & $\begin{array}{l}\text { Ring e Van de Ven (1994); } \\
\text { Rousseau et al. (1998); } \\
\text { Chen, Lin e Yen (2014). }\end{array}$ \\
\hline \multirow{2}{*}{ Desempenho Organizacional } & Desempenho financeiro (DESFIN) & $\begin{array}{l}\text { Burnes e New (1997); } \\
\text { Comb e Ketchen (1999); } \\
\text { Vereecke e Muylle (2006); } \\
\text { Palmatier, Dant e Grewal (2007). }\end{array}$ \\
\cline { 2 - 3 } & Desempenho não-financeiro (DESNFIN) & $\begin{array}{l}\text { Selnes e Sallis (2003); } \\
\text { Beugelsdijk, Koen e Noorderhaven (2009); } \\
\text { Ashnai et al. (2016). }\end{array}$ \\
\cline { 2 - 3 } & &
\end{tabular}

Fonte: Elaboração própria.

Para análise dos dados utilizaram-se técnicas de estatística descritiva e modelagem de equações estruturais, por meio do Smart PLS, versão 3.0. Conforme Hair Jr et al. (2009), a Modelagem de Equações Estruturais (SEM) é adequada quando se anseia incorporar determinadas variáveis latentes na análise, e quando as relações de dependência e independência entre as variáveis são objeto de investigação. A SEM foi estimada a partir dos Mínimos Quadrados Parciais (Partial Least Squares - PLS), em que é possível analisar modelos formativos com amostras menores (CHIN; NEWSTED, 1999) e com possíveis outliers (CHIQUOINE; HJALMARSSON, 2009). Assim, pode-se analisar as variáveis de forma isolada e, na sequência, calcular as relações de causalidade.

\section{ANÁLISE E DISCUSSÃO DOS RESULTADOS}

\subsection{Perfil dos respondentes e das empresas franqueadas}

O perfil dos respondentes (gênero, idade, cargo, nível de escolaridade e tempo que trabalha na função) é apresentado na Tabela 1.

Observa-se na Tabela 1, que do total de 151 respondentes, a maioria é do gênero masculino, com predominância nas faixas etárias até 40 anos, maior concentração nos cargos de proprietário, e grande parte tem como maior nível de escolaridade curso de graduação. Depreende-se que a gestão destas empresas é realizada, na sua maioria, por profissionais do gênero masculino, dentro de uma faixa etária relativamente jovem, com um quadro restrito de funcionários e com indicativo de que seguem a regulação do setor quanto à presença de profissionais com ensino superior, no caso da área farmacêutica. 
Tabela 1 Perfil dos respondentes da pesquisa

\begin{tabular}{|c|c|c|}
\hline Gênero & Quant. & $\%$ \\
\hline Feminino & 65 & 43,05 \\
\hline Masculino & 86 & 57,95 \\
\hline Total & 151 & $100 \%$ \\
\hline Idade & Quant. & $\%$ \\
\hline 20 a 30 anos & 45 & 29,80 \\
\hline 31 a 40 anos & 59 & 39,07 \\
\hline 41 a 50 anos & 32 & 21,19 \\
\hline 51 a 60 anos & 14 & 9,27 \\
\hline acima de 60 anos & 1 & 0,66 \\
\hline Total & 151 & $100 \%$ \\
\hline Média de idade & & 37 \\
\hline Desvio padrão da idade & & 8,81 \\
\hline Cargo/função & Quant. & $\%$ \\
\hline Proprietário & 75 & 37,69 \\
\hline Gerente & 55 & 27,64 \\
\hline Farmacêutico & 54 & 27,14 \\
\hline Administrativo & 15 & 7,54 \\
\hline
\end{tabular}

* No cago/função, o total ultrapassa o número de respondentes devido a indicação de mais de uma alternativa.

\begin{tabular}{l|c|c}
\hline \multicolumn{1}{c|}{ Nível de Escolaridade } & Quant. & \% \\
\hline Ensino fundamental & 3 & 1,99 \\
\hline Ensino médio & 33 & 21,85 \\
\hline Graduação & 106 & 70,20 \\
\hline Especialização & 9 & 5,96 \\
\hline Total & $\mathbf{1 5 1}$ & $\mathbf{1 0 0 \%}$
\end{tabular}

Fonte: Dados da pesquisa.

A caracterização das empresas pesquisadas é apresentada na Tabela 2, em específico o tempo que a empresa trabalha sob a forma de franquia, o tempo que trabalha com o franqueador atual e qual a franquia, além da localização da unidade franqueada.
Tabela 2 Caracterização das empresas franqueadas

\begin{tabular}{|c|c|c|}
\hline Tempo em Franquia & Quant. & $\%$ \\
\hline até 5 anos & 66 & 43,71 \\
\hline 6 a 10 anos & 55 & 36,42 \\
\hline 11 a 15 anos & 18 & 11,92 \\
\hline 16 a 20 anos & 11 & 7,28 \\
\hline acima de 21 anos & 1 & 0,66 \\
\hline Total & 151 & $100 \%$ \\
\hline Média de tempo em franquia & & 7 \\
\hline Desvio padrão do tempo em franquia & & 5,02 \\
\hline Franquia & Quant. & $\%$ \\
\hline Farmais & 11 & 7,28 \\
\hline Farmatotal & 42 & 27,81 \\
\hline Forte Farma & 10 & 6,62 \\
\hline Hiper Farma & 47 & 31,13 \\
\hline Master Farma & 10 & 6,62 \\
\hline Maxifarma & 29 & 19,21 \\
\hline Medfarma & 2 & 1,32 \\
\hline Total & 151 & $100 \%$ \\
\hline
\end{tabular}

\begin{tabular}{l|c|c}
\multicolumn{1}{c|}{ Tempo na franquia atual } & Quant. & $\%$ \\
\hline até 5 anos & 88 & 58,28 \\
\hline 6 a 10 anos & 48 & 31,79 \\
\hline 11 a 15 anos & 11 & 7,28 \\
\hline 16 a 20 anos & 4 & 2,65 \\
\hline acima de 21 anos & 0 & 0,00 \\
\hline Total & $\mathbf{1 5 1}$ & $\mathbf{1 0 0 \%}$ \\
\hline Média de tempo na franquia atual & & 6 \\
\hline Desvio padrão do tempo na franquia atual & & 4,21 \\
\hline \multicolumn{1}{|c|}{ Localização da Franqueada } & Quant. & $\%$ \\
\hline Curitiba & 112 & 74,17 \\
\hline Região Metropolitana de Curitiba & 32 & 21,19 \\
\hline Interior do Estado do Paraná & 7 & 4,64 \\
\hline Total & $\mathbf{1 5 1}$ & $\mathbf{1 0 0 \%}$
\end{tabular}

Fonte: Dados da pesquisa. 
Conforme a Tabela 2, a maioria das empresas da amostra indicou experiência em franquias de farmácias nas duas faixas inferiores, até 10 anos, o que pode sugerir que o sistema de franquias do setor nessa região é relativamente novo. A amostra, em sua maioria, é de Curitiba, PR, o que contribui para a análise da realidade local e do seu entorno. Embora esses dados não possam ser generalizados, podem apresentar semelhanças com os de outras regiões. Três franquias mais se destacaram na amostra: Hiper Farma, Farmatotal e Maxifarma.

A Hiper Farma e a Master Farma possuem como denominação jurídica rede associativista. Para desvendar esse aspecto acessaram-se documentos de franqueados, a fim de realizar uma análise do contrato firmado entre as farmácias e a rede, além dos propósitos declarados em seus websites. Notou-se que os objetivos comerciais são similares quando comparados com outras redes. A participação das lojas ocorre em ambas as franquias, o poder de decisão é equivalente a outras franquias, pois mesmo não sendo associativistas também proporcionam espaço para melhorias. Considerando os propósitos desta pesquisa, optou-se por mantê-las na amostra, visto suas similaridades com as demais.

Depreende-se do perfil dos respondentes e dos franqueados que o acúmulo de funções dos gestores das franqueadas deve-se principalmente ao fato de se tratar de arranjos comerciais com baixa complexidade, o que lhes permite desempenhar múltiplas atividades, consequentemente, costumam conhecer em profundidade o seu negócio. Assim, presume-se que reúnem as condições necessárias para contribuir com esta pesquisa.

\subsection{Modelo de mensuração}

Um construto não pode ser diretamente mensurado, mas sim por uma ou mais de suas variáveis. Neste estudo, têm-se como variáveis latentes as dimensões da Teoria da Cooperação (resolução conjunta de problemas, uso do poder e disposição para adaptação à mudanças). Além dessas, o compartilhamento de informações e a confiança interorganizacional são variáveis latentes na posição de variáveis mediadoras. O modelo estrutural indica ainda como variáveis latentes o desempenho financeiro e não financeiro.

No modelo de mensuração verifica-se a validade convergente, a confiabilidade composta e do indicador e a validade discriminante (HAIR JR et al., 2009). A validade convergente toma como base o acompanhamento da Variância Média Extraída (Average Variance Extracted - AVE), que deve ser maior que 0,50 (HAIR JR et al., 2009), e representa a quantidade de variância compartilhada entre os indicadores de cada variável latente. Na confiabilidade interna dos construtos, o alfa de Cronbach deve estar acima de 0,70 , mas valores inferiores são aceitáveis nas Ciências Sociais; e nos testes da confiabilidade composta, os valores de 0,70 e 0,90 são considerados satisfatórios (HAIR JR et al., 2009). Na validade discriminante, o teste foi realizado pela observação das cargas cruzadas (Cross Loading). Na Tabela 3 apresentam-se os resultados dos testes da validade do modelo estrutural.

Tabela 3 Validade do modelo estrutural

\begin{tabular}{|c|c|c|c|c|c|c|c|c|c|}
\hline \multirow{2}{*}{ Descrição } & \multirow{2}{*}{$\begin{array}{c}\text { Confiab. } \\
\text { Composta } \\
>0,70\end{array}$} & \multirow{2}{*}{$\begin{array}{c}\text { Alfa de } \\
\text { Cronbach } \\
>0,70\end{array}$} & \multirow{2}{*}{$\begin{array}{c}\text { AVE } \\
>0,50\end{array}$} & \multicolumn{6}{|c|}{ Validade Discriminante } \\
\hline & & & & 1 & 2 & 3 & 4 & 5 & 6 \\
\hline 1. Compartilham. de informações & 0,896 & 0,825 & 0,741 & 0,861 & & & & & \\
\hline 2. Confiança Interorganizacional & 0,953 & 0,940 & 0,773 & 0,743 & 0,879 & & & & \\
\hline 3. Desempenho Financeiro & 0,931 & 0,901 & 0,771 & 0,797 & 0,831 & 0,878 & & & \\
\hline 4. Desempenho não Financeiro & 0,933 & 0,904 & 0,777 & 0,822 & 0,763 & 0,840 & 0,881 & & \\
\hline 5. Resolução conjunta de problemas & 0,819 & 0,712 & 0,535 & 0,574 & 0,641 & 0,555 & 0,615 & 0,731 & \\
\hline 6. Uso do Poder & 0,931 & 0,901 & 0,772 & 0,545 & 0,551 & 0,487 & 0,539 & 0,518 & 0,879 \\
\hline
\end{tabular}

Fonte: Dados da pesquisa. 
Fonte: Dados da pesquisa.

A AVE demonstrou que as cargas fatoriais de cinco indicadores não apresentaram confiabilidade individual aceitável $(0,50)$, assim optou-se pela sua exclusão. Após cada exclusão foram refeitos os testes estatísticos, até a adequação do modelo. Cabe ressaltar que após a exclusão da terceira variável relativa à disposição para adaptação à mudanças, optou-se pela exclusão desta dimensão, pois a validade desta variável latente poderia estar prejudicada com a sua mensuração por apenas dois indicadores.

$\mathrm{Na}$ análise da confiabilidade interna dos construtos, o alfa de Cronbach apresentou as cargas dos indicadores frente aos seus respectivos construtos, sendo que todas as variáveis apresentaram correlações fortes ou moderadas com seu construto correspondente. Da mesma forma, a confiabilidade composta dos construtos mostrou que nenhuma das variáveis possui valor inferior a 0,80 , indicando alta confiabilidade interna.

No estudo de Mahama (2006), compartilhamento de informações apresentou um alfa de 0,796 , como parte do construto da Teoria da Cooperação. Nesta pesquisa, essa formou uma variável mediadora entre cooperação e desempenho e apresentou um alfa de 0,825, superior ao de Mahama (2006), corroborando assim com o modelo proposto nesta pesquisa.

A validade discriminante do modelo teórico mostrou que cada variável latente possui cargas maiores que seus respectivos construtos teóricos, ou seja, as cargas fatoriais calculadas dentro de cada construto de origem para seus respectivos indicadores são mais elevadas, comparativamente aos demais construtos do modelo. Tal sugere que as questões utilizadas são, de fato, indicadores observáveis dos construtos estabelecidos.

Denota-se que o modelo de mensuração está ajustado, com significância estatística de seus caminhos obtidos pela validade convergente, validade discriminante e confiabilidade. Esses resultados indicam validade preditiva adequada e permite analisar o modelo estrutural.

\subsection{Modelo estrutural}

No modelo estrutural são analisadas os caminhos (Path), em que se verifica a relação estabelecida entre dois construtos e sua significância estatística (HAIR JR et al., 2009). Após a geração dos coeficientes de caminho, efetua-se o cálculo da distribuição $t$ de Student para verificar as relações significantes entre as variáveis do modelo estrutural. Na Tabela 4, evidenciam-se as relações dos coeficientes estimados dos caminhos do modelo estrutural e níveis de significância, conforme as hipóteses H1a, H1b, H2 e H3, exceto as de mediação.

Os valores do coeficiente de determinação $\mathrm{R}^{2}$ indicam que os construtos do compartilhamento de informações e da confiança interorganizacional são explicados, respectivamente, em $41,3 \%$ e $47,7 \%$, pelas variáveis da cooperação que compõem o modelo (resolução conjunta de problemas e uso do poder). E que $76,4 \%$ do desempenho financeiro e $73,7 \%$ do desempenho não financeiro são explicados pelo papel mediador das variáveis latentes confiança interorganizacional e compartilhamento de informações, em relação às dimensões da cooperação.

Desta forma, infere-se que o desempenho pode ser explicado pelas duas dimensões da cooperação (resolução conjunta de problemas e uso do poder) e pela confiança interorganizacional e compartilhamento de informações. $\mathrm{O}$ valor $\mathrm{R}^{2}$ dos construtos desta pesquisa é superior ao do estudo de Mahama (2006), com um valor de 56\% de explicação para o desempenho, considerando dimensões semelhantes para a cooperação.

$\mathrm{Na}$ análise das hipóteses da pesquisa, no caso da hipótese $\mathrm{H} 1 \mathrm{a}$, que previa relação positiva entre a cooperação em franquia e o desempenho financeiro do franqueado, os resultados não indicaram significância estatística para tal relação direta, assim não se aceita a H1a. A hipótese H1b, que previa relação positiva com o desempenho não financeiro, também não apresentou significância, logo, não se aceita a H1b. 
Tabela 4 Resultados do modelo estrutural - Efeitos diretos

\begin{tabular}{c|l|c|c|c|c|c|c}
\hline \multicolumn{1}{c|}{ Hipóteses } & Coef. $\boldsymbol{\beta}$ & $\begin{array}{c}\text { Desvio } \\
\text { padrão }\end{array}$ & $\begin{array}{c}\text { Erro } \\
\text { padrão }\end{array}$ & Valor t & Valor p & Decisão \\
\hline H1a & $\begin{array}{l}\text { Resolução conjunta de problemas } \rightarrow \\
\text { Desempenho financeiro }\end{array}$ & $-0,021$ & $-0,016$ & 0,064 & 0,332 & 0,7399 & Não aceita \\
\hline H1a & Uso do poder $\rightarrow$ Desempenho financeiro & $-0,034$ & $-0,028$ & 0,061 & 0,555 & 0,5788 & Não aceita \\
\hline H1b & $\begin{array}{l}\text { Resolução conjunta de problemas } \rightarrow \\
\text { Desempenho não financeiro }\end{array}$ & 0,115 & 0,120 & 0,063 & 1,818 & 0,0693 & Não aceita \\
\hline H1b & $\begin{array}{l}\text { Uso do poder } \rightarrow \\
\text { Desempenho não financeiro }\end{array}$ & 0,040 & 0,043 & 0,065 & 0,606 & 0,5445 & Não aceita \\
\hline H2 & $\begin{array}{l}\text { Resolução conjunta de problemas } \rightarrow \\
\text { Compartilhamento de informações }\end{array}$ & 0,398 & 0,397 & 0,079 & 5,060 & 0,0000 & Aceita \\
\hline Uso do poder $\rightarrow$ \\
Compartilhamento de informações
\end{tabular}

$\mathbf{R}^{2}$ : Compartilhamento de informações $=0,413$; Confiança Interorganizacional = 0,477; Desempenho Financeiro =0,737; Desempenho não Financeiro $=0,764$; Resolução conjunta de problemas $=0,000$; Uso do Poder $=0,000$.

A comparação com o estudo de Mahama (2006), que foi utilizado como base para as indagações acerca dos componentes da Teoria a Cooperação, fica prejudicada, devido ao fato dele ter utilizado as quatro dimensões da cooperação propostas por Heide e Miner (1992) - compartilhamento de informações, resolução conjunta de problemas, uso do poder e disposição para adaptação à mudanças - em um único construto. No estudo de Mahama (2006), três dimensões da cooperação (resolução conjunta de problemas, uso do poder e disposição para adaptação à mudanças) apresentaram-se estatisticamente significantes quando relacionadas ao desempenho organizacional.

A hipótese $\mathrm{H} 2$ previa relação entre cooperação em franquia e compartilhamento de informações, sendo que os resultados encontrados foram positivos e significativos, assim encontra-se suporte para aceitar a H2. Portanto, a relação das dimensões da cooperação (resolução conjunta de problemas e uso do poder) com o compartilhamento de informações sugere que essa dimensão pode ser abordada de maneira isolada. Em alguns casos pode apresentar forte poder de explicação para as demais dimensões da Teoria da Cooperação, como no presente estudo, portanto, diferente dos estudos de Heide e Miner (1992) e Mahama (2006), que a consideraram como uma dimensão do construto da Teoria da Cooperação.

A hipótese $\mathrm{H} 3$ previa relação positiva entre cooperação em franquia e confiança interorganizacional. Os resultados foram significativos ao nível de 5\%, o que permite aceitar a H3. Embora o impacto direto da confiança no desempenho seja amplamente abordado nos estudos da área, pouco se sabe acerca dos efeitos da confiança vista como mediadora (JIANG et al., 2015). As análises posteriores abordam essa relação da confiança como papel mediador.

As hipóteses de mediação foram analisadas com base nos resultados apresentados na Tabela 5, que evidencia o teste $t$ e o valor $p$, pela mediação da confiança interorganizacional e do compartilhamento de informações. As verificações também se valeram de valores obtidos a partir do Bootstrapping, que confirmaram as hipóteses de mediação. Para que a mediação fosse confirmada como completa, as relações analisadas pelos testes de Sobel, Aroian e Goodman deveriam apresentar $\mathrm{p}<0,05$, e a relação direta extraída do Bootstrapping deveria ser não significativa (HAIR JR et al., 2009). 
Tabela 5 Resultados do modelo estrutural - Efeitos indiretos

\begin{tabular}{|c|c|c|c|c|}
\hline & Hipóteses & Teste $\mathbf{t}$ & Valor $\mathbf{p}$ & Decisão \\
\hline \multirow{2}{*}{$\mathrm{H} 4 \mathrm{a}$} & $\begin{array}{l}\text { Resolução conjunta de problemas } \rightarrow \text { Compartilhamento de informações } \rightarrow \\
\text { Desempenho financeiro }\end{array}$ & 0,332 & 0,7399 & Aceita \\
\hline & UsoPoder $\rightarrow$ Compartilhamento de informações $\rightarrow$ Desempenho financeiro & 0,555 & 0,5788 & Aceita \\
\hline \multirow{2}{*}{$\mathrm{H} 4 \mathrm{~b}$} & $\begin{array}{l}\text { Resolução conjunta de problemas } \rightarrow \text { Compartilhamento de informações } \rightarrow \\
\text { Desempenho não Financeiro }\end{array}$ & 1,818 & 0,0693 & Aceita \\
\hline & $\begin{array}{l}\text { UsoPoder } \rightarrow \text { Compartilhamento de informações } \rightarrow \\
\text { Desempenho não Financeiro }\end{array}$ & 0,606 & 0,5445 & Aceita \\
\hline \multirow{2}{*}{$\mathrm{H} 5 \mathrm{a}$} & $\begin{array}{l}\text { Resolução conjunta de problemas } \rightarrow \text { Confiança interorganizacional } \rightarrow \text { Desem- } \\
\text { penho financeiro }\end{array}$ & 0,332 & 0,7399 & Aceita \\
\hline & Uso do Poder $\rightarrow$ Confiança interorganizacional $\rightarrow$ Desempenho financeiro & 0,555 & 0,5788 & Aceita \\
\hline \multirow{2}{*}{$\mathrm{H} 5 \mathrm{~b}$} & $\begin{array}{l}\text { Resolução conjunta de problemas } \rightarrow \text { Confiança interorganizacional } \rightarrow \text { Desem- } \\
\text { penho não Financeiro }\end{array}$ & 1,818 & 0,0693 & Aceita \\
\hline & $\begin{array}{l}\text { Uso do Poder } \rightarrow \text { Confiança interorganizacional } \rightarrow \\
\text { Desempenho não Financeiro }\end{array}$ & 0,606 & 0,5445 & Aceita \\
\hline
\end{tabular}

Fonte: Dados da pesquisa.

As hipóteses H4a e H4b previam relação entre cooperação em franquias e o desempenho financeiro e não financeiro, mediada pelo compartilhamento de informações. Os resultados convergem para a aceitação de ambas as hipóteses, pois os franqueados percebem melhora do desempenho em ambientes de cooperação e trocas informacionais, corroborando com o proposto no estudo de Jiang et al. (2015), que apontavam um possível caminho com os efeitos indiretos do compartilhamento de informações.

Quando a cooperação em franquia é mediada pela confiança interorganizacional, o desempenho financeiro do franqueado é alterado. Contudo, o coeficiente de efeito diminuiu e suporta a aceitação da hipótese H5a, corroborado com os índices apresentados pelos testes de Sobel, Aroian e Goodman (HAIR JR et al., 2009). Infere-se que os respondentes do estudo percebem melhora no desempenho financeiro, quando há confiança interorganizacional.

$\mathrm{Na}$ amostra, a confiança interorganizacional pode ser um atenuante para os comportamentos cooperativos, o que sugere que o franqueado, a partir de uma atitude positiva em relação ao franqueador, pode melhorar seu desempenho. Tal corrobora os achados de Jiang et al. (2015), que avaliaram a confiança como mediadora em relações com variáveis dependentes de desempenho.
Outro fator que pode afetar os comportamentos cooperativos é a sua relação com o desempenho não financeiro, mediada pela confiança interorganizacional. Os resultados dos testes realizados suportam a aceitação da H5b, pois as relações positivas encontradas evidenciam ser uma dimensão de caminho importante para o desempenho não financeiro. Das e Teng (1998) reforçam que, ao invés de focar apenas em resultados unidimensionais relacionados à confiança interorganizacional, ela pode ser um consistente argumento para a melhora percebida do desempenho, em consonância com a Teoria da Cooperação.

Cannon e Perreault (1999) argumentam que, embora a partilha de informações possa, potencialmente, melhorar o desempenho, ela também abre caminho para comportamentos oportunistas, com implicações negativas no desempenho. Mayer, Davis e Schoorman (1995) advertem que não é a quantidade de informações compartilhadas que resulta em desempenho superior, mas a sua qualidade e relevância.

\subsection{Discussão dos resultados}

As dimensões da cooperação (resolução conjunta de problemas e uso do poder) não apresentaram significância estatística direta com o desempenho financeiro e não financeiro. Apesar dessas interações 
não apresentarem significância, elas corroboram as mediações propostas. Esperava-se que essas relações diretas não fossem significativas, ainda que Heide e Miner (1992) e Mahama (2006) tenham encontrado significância, mas a amostra era diferente e as interações propostas também. Embora uma parte extensa do estudo empírico tenha focado na natureza e estrutura das relações de franquia, o papel dos fatores comportamentais dos franqueados não pode ser totalmente compreendido, pois trata-se de uma abordagem com complexidades não resolvidas (NYADZAYO; MATANDA; EWING, 2016).

Por sua vez, as relações entre as dimensões da cooperação (resolução conjunta de problemas e uso do poder) apresentaram significância com as variáveis mediadoras do estudo (confiança interorganizacional e compartilhamento de informações). Esses achados apontam para o poder mediador desses construtos. A significância estatística, pelo teste $t$, das variáveis mediadoras com o desempenho reafirma a explicação estatística de que a cooperação, aliada ao compartilhamento de informações e a confiança interorganizacional, pode sugerir uma melhora percebida no desempenho. Esse resultado encontra apoio nos estudos que sugerem uma possível explicação das trocas interfirmas, alicerçadas em ambientes de relacionamento de confiança, buscando melhoras no desempenho (CASIDY; NYADZAYO, 2017).

Como as relações de cooperação visam alavancar os negócios das empresas (ANDERSON; NARUS, 1990), e o relacionamento interorganizacional deve ser fundamentado em fatores que direcionem a percepção de melhora econômica (JIANG et al., 2016), elementos como a confiança interorganizacional (RING; VAN DE VEN, 1994) e o compartilhamento de informações (MAHAMA, 2006) traduzem um nexo de causalidade. Nesta pesquisa as relações diretas entre as dimensões da cooperação e o desempenho não apresentaram significância estatística. Para verificar o efeito total das relações entre as variáveis, analisaram-se as relações entre as variáveis de forma integrada. Como o modelo teórico da pesquisa contêm variáveis de mediação, verificou-se se a mediação influencia na relação entre as variáveis dependente e independente.

Cannon e Perreault (1999) argumentam que a cooperação não implica apenas na aquiescência das partes para as necessidades do outro, mas sim, num trabalho harmoniosamente colaborativo para suprir as necessidades do outro. Mesmo que a motivação para a opção de franquias seja por pressões ambientais (KASTNER et al., 2019), esse modelo requer que ambas as partes confluam para a resolução conjunta de problemas, como uma responsabilidade conjunta, pois quando os esforços não são coordenados e os problemas são vistos de forma isolada, tem-se um baixo comportamento cooperativo (MAHAMA, 2006). Essas condições implicam que as partes são, ou não, capazes de negociar soluções quando surgem problemas (HEIDE; MINNER, 1992).

Embora o poder esteja presente nas relações de troca (MAHAMA, 2006), ele existirá de maneira diferente para as partes, sendo que uma forma de suavizar os conflitos provocados são as relações contratuais formais, que delimitam as responsabilidades entre as partes. O conhecimento das normas estabelecidas em contrato com a franqueadora pode ser reforçado com o compartilhamento de informações, em detrimento de um contrato firmado mais de cunho jurídico do que um instrumento utilizado nas relações. Pode ainda identificar a vulnerabilidade por comportamentos oportunistas, cabendo assim o seu uso ou não, a fim de minimizar oportunidades de extração de vantagens (HEIDE; MINER, 1992). Contudo, destaca-se a importância do compartilhamento de conhecimento entre as partes, pois o esforço destas interações é percebido empiricamente no desempenho (LEE, 2017).

Das e Teng (1998) afirmam que a confiança mutua entre os parceiros é um fator essencial para acordos de cooperação, conforme observado nesta pesquisa. Os resultados da pesquisa corroboram com a presunção de que a confiança dos franqueados em relação ao franqueador é importante, pois afeta o desempenho percebido (CHIOU; DROGE, 2015). Em relação ao compartilhamento de informações, esse é visto como benéfico ao relacionamento interfirmas. Reduzida partilha de recursos pode indicar que uma das partes não quer fornecer os recursos necessários para a continuidade do relacionamento, sinalizando comportamentos oportunistas e falta de confiança na outra parte, o que pode ser prejudicial ao desempenho de ambas as partes (JIANG et al., 2015).

Experiências anteriores de cooperação e reputação dos parceiros são apontadas como componentes 
importantes nas interações interfirmas quando se objetiva melhora no desempenho (ANAND; KHANNA, 2000). Os resultados da pesquisa indicam que a melhora do desempenho organizacional, tanto o financeiro como o não financeiro, também pode decorrer de comportamentos cooperativos entre as empresas, conforme já apontado por Heide e Miner (1992), Smith, Carrol e Ashford (1995), Das e Teng (1998), Cannon e Perreault (1999), Mahama (2006), Beugelsdijik, Koen e Noorderhaven (2009) e Ashnai et al. (2016).

\section{CONSIDERAÇÕES FINAIS}

As postulações teóricas e as evidências empíricas deste estudo permitiram analisar as hipóteses formuladas. A H1 não foi aceita, por não mostrar significância estatística na relação direta entre a cooperação e o desempenho financeiro e não financeiro. Isso sugere que os comportamentos cooperativos não são claramente percebidos como um fator que influencia diretamente o desempenho. Esse resultado difere do estudo de Mahama (2006), onde houve significância estatística para três dimensões da cooperação (resolução conjunta de problemas, uso do poder e disposição para adaptação à mudanças) em relação direta com o desempenho. As hipóteses $\mathrm{H} 2$ e H3 foram aceitas, indicando que a cooperação tem relação positiva e significativa com compartilhamento de informações e confiança interorganizacional.

As mediações propostas pelas hipóteses $\mathrm{H} 4$ e H5 foram confirmadas, ou seja, o compartilhamento de informações e a confiança interorganizacional exercem papel mediador nas relações entre a cooperação e o desempenho organizacional. O compartilhamento de informações apresentou efeito mediador entre a cooperação (resolução conjunta de problemas e uso do poder) e o desempenho, consistente com Gruenfeld et al. (1996). Já a confiança interorganizacional revelou ser importante para o desempenho organizacional (ROUSSEAU et al., 1998), pois o aumento da confiança gera um clima organizacional favorável (CONDREY, 1995) e pode exercer papel mediador na melhora do desempenho.

Os resultados da pesquisa mostraram que o desempenho financeiro e não financeiro é afetado pela presença da confiança interorganizacional e pelo compartilhamento de informações. E as duas dimensões da cooperação (resolução conjunta de problemas e uso do poder) possuem efeito direto menor sobre o desempenho em comparação ao efeito indireto, o que sugere que as variáveis mediadoras são representativas da amostra pesquisada. Assim, conclui-se, que o compartilhamento de informações e a confiança interorganizacional são questões complexas edificadas em relações interfirmas, e nesta pesquisa revelaram-se um importante nexo de causalidade entre a cooperação e o desempenho.

Este estudo contribui para a literatura ao demonstrar a importância do compartilhamento de informações e da confiança interorganizacional para estimular comportamentos cooperativos com vistas na melhora do desempenho. Os resultados ainda destacam que quando esses fatores mediadores (confiança interorganizacional e compartilhamento de informações) não são salientados, a relação direta entre a cooperação e o desempenho organizacional não é fortemente percebida. Estudos, como o de Fukuyama (1996), propõem que uma relação interorganizacional baseada na confiança pode sugerir maior cooperação espontânea por parte dos envolvidos, e isso pode influenciar positivamente no desempenho organizacional (PARTIMATIER; DANT; GREWAL, 2007).

As interações investigadas denotam que o desempenho organizacional é um consequente que favorece a compreensão do papel destes construtos na prática organizacional e influencia positivamente nos comportamentos cooperativos, conjuntamente com a confiança interorganizacional. Estes resultados são relevantes para os franqueados que buscam maneiras de melhorar o seu relacionamento interfirmas, visto que não é suficiente ter esforços coordenados no intuito de cooperar para atingir melhora no desempenho, é fundamental que nessa relação interorganizacional haja compartilhamento de informações e confiança.

Diante das limitações deste estudo, recomenda-se que futuras pesquisas verifiquem a validade dos construtos em outros ambientes institucionais. No entanto, outras variáveis podem ser incluídas, de modo a contribuir para o modelo testado. A inclusão de variáveis de controle também pode ser interessante no estudo, por exemplo, franquia, localização, número de funcionários, entre outros. Esta pesquisa foi 
projetada para capturar as trocas informacionais, tais como praticadas, portanto, não se examinou explicitamente a qualidade das informações compartilhadas, o que pode ser foco de outra pesquisa.

\section{REFÊRENCIAS}

ABRADE, E. Cooperação interorganizacional: Fonte de aprendizagem e vantagem competitiva ou oportunismo? Revista de Administração da UNIMEP, v. 8, n. 2, p. 154-179, 2010.

ANAND. B. N.; KHANNA, T. Do firms learn to create value? The case of aliances. Strategic Managemente Journal, v. 21, n. 3, p. 295-315, 2000.

ANDERSON, C.; THOMSPON. L. L. Affect from the top down: how powerful individuals' positive affect shapes negotiations. Organizational Behavior and Human Decision Processes, v. 95, n. 2, p. 125-139, 2004.

ANDERSON, E.; WEITZ, B. The use of pledges to build and sustain commitment in distribution channels. Journal of Marketing Research, v. 29, n. 1, p. 18-34, 1992.

Anderson, J.; Narus, J. A model of distributor firm and manufacturer firm working partnership. Journal of Marketing Research, v. 54, n. 1, p. 42-58, 1990.

ANTHONY, R.N.; GOVINDARAJAN, V.; DEARDEN, J. Management control systems. 9. ed. Boston: Irwin McGraw-Hill, 1998.

ASHNAI, B.; HENNEBERG, S. C.; NAUDÉ, P.; FRANCESCUCCI, A. Inter-personal and inter-organizational trust in business relationships: An attitudebehavior-outcome model. Industrial Marketing Management, v. 52, n. 1, p. 128-139, 2016.

ATALLAH, G. Information sharing and the stability of cooperation in research joint ventures. Economics of Innovation and New Technology, v. 12, n. 6, p. 531-554, 2003.
AXELROD, R. The evolution of cooperation. New York: Basic Books, 1984.

BACHMANN, R. Trust, power and control in trans-organizational relations. Organization Studies, v. 22 , n. 2, p. 337-365, 2001.

BALESTRIN, A.; VERSCHOORE, J. R.; REYES JR, E. O campo de estudos sobre redes de cooperação interorganizacional no Brasil. Revista de Administração Contemporânea, v. 14, n. 3, p. 458-477, 2010.

BARNARD, C. The functions of the executive. Cambridge, MA: Harvard University Press, 1938.

\section{BEUGELSDIJK, S.; KOEN, C.; NOORDERHAVEN,} N. A dyadic approach to the impact of differences in organizational culture on relationship performance. Industrial Marketing Management, v. 38, n. 3, p. 312-323, 2009.

BEUREN, I.M.; DAL VESCO, D.G. Management control systems and performance in strategic supply relationships. International Journal of Productivity and Performance Management, ahead-of-print, 2021.

BRITO, L. A. L.; BRITO, E. P. Z.; HASHIBA, L. H. What type of cooperation with suppliers and customers leads to superior performance? Journal of Business Research, v. 67, n. 5, p. 952-959, 2014.

BROWNING, L. D.; BEYER, J. M.; SHETLER, J. C. Building cooperation in a competitive industry: Sematech and the semiconductor industry. Academy of Management Journal, v. 38, n. 1, p. 113-151, 1995.

BURNES, B.; NEW, S. Collaboration in customer囚supplier relationships: strategy, operations and the function of rhetoric. International Journal of Purchasing and Materials Management, v. 33, n. 3, p. 10-17, 1997.

CANNON, J. P.; PERREAULT, W. D. Buyer-seller relationships in business markets. Journal of Marketing Research, v. 36, n. 4, p. 439-460, 1999. 
CARDINAELS, E.; VAN VEEN-DIRKS, P.M.G. Financial versus non-financial information: The impact of information organization and presentation in a Balanced Scorecard. Accounting, Organizations and Society, v. 35, n. 6, p. 565$578,2010$.

CASIDY, R.; NYADZAYO, M. Drivers and outcomes of relationship quality with professional service firms: An SME owner-manager perspective. Industrial Marketing Management, in press, 2017. Doi: https://doi.org/10.1016/j. indmarman.2017.09.011

CHEN, C. C.; CHEN, X. P.; MEINDL, J. R. How can cooperation be fostered? The cultural effects of individualism-collectivism. Academy of Management Review, v. 23, n. 2, p. 285-304, 1998.

CHEN, Y. H.; LIN, T.-P.; YEN, D. C. How to facilitate inter-organizational knowledge sharing: The impact of trust. Information \& Management, v. 51, n. 5, p. 568-578, 2014.

CHIN, W. W.; NEWSTED, P. R. Structural equation modelling analysis with small samples using partial least squares. In: HOYLE, R. H. (ed.). Statistical strategies for small sample research. Thousand Oak: Sage Publications, 1999.

CHIOU, J. S.; DROGE C. The effects of standardization and trust on franchisee's performance and satisfaction: A study on franchise systems in the growth stage. Journal of Small Business Management, v. 53, n. 1, p. 129-144, 2015.

CHIQUOINE, B.; HJALMARSSON, E. Jackknifing stock return predictions. Journal of Empirical Finance, v. 16, n. 5, p. 793-803, 2009.

COMBS, J. G.; KETCHEN, D. J. Explaining interfirm cooperation and performance: toward a reconciliation of predictions from the resource-based view and organizational economics. Strategic Management Journal, v. 20, n. 9, p. 867-888, 1999.
COMBS, K. L. The role of information sharing in cooperative research and development. International Journal of Industrial Organization, v. 11, n. 4, p. 535-551, 1993.

CONDREY, S. Reforming human resource management systems: exploring the importance of organizational trust. American Review of Public Administration, v. 25, n. 4, p. 341-350, 1995.

DAS, T. K.; TENG, B. S. Between trust and control: Developing confidence in partner cooperation in alliances. Academy of Management Review, v. 23, n. 3, p. 491-512, 1998.

DEKKER, H.C.; DING, R.; GROOT, T. Collaborative performance management in interfirm relationships. Journal of Management Accounting Research, v. 28, n. 3, p. 25-48, 2016.

DEUTSCH, M. A theory of cooperation and competition. Human Relations, v. 2, n. 1, p. 129-152, 1949.

FUKUYAMA, F. Confiança: As virtudes sociais e a criação da prosperidade. Rio de Janeiro: Rocco, 1996.

GAMBETTA, D. Trust: Making and breaking cooperative relations. New York: Basil Blackwell, 1988.

GRUENFELD, D. H.; MANNIX, E. A.; WILLIAMS, K. Y.; NEALE, M. A. Group composition and decision-making: How member familiarity and information distribution affects process and performance. Organizational Behavior Human Decision Processes, v. 67, n. 1, p. 1-15, 1996.

GULATI, R. Does familiarity breed trust? The implications of repeated ties for contractual choice in alliances. Academy of Management Journal, v. 38, n. 1, p. 85-112, 1995. 
HAIR JR, J. F.; BLACK, W. C.; BABIN, B. J.; ANDERSON, R. E.; TATHAM, R. L. Análise multivariada de dados. 6. ed. Porto Alegre: Bookman, 2009.

HEIDE, J. B.; JOHN, G. Do norms matter in marketing relationships? The Journal of Marketing, v. 56, n. 1, p. 32-44, 1990.

HEIDE, J. B.; MINER, A. S. The shadow of the future: Effects of anticipated interaction and frequency of contact on buyer-seller cooperation. Academy of Management Journal, v. 35, n. 2, p. 265-291, 1992.

HERZ, M.; HUTZINGER, C.; SEFERAGIC, H.; WINDSPERGER, J. Trust, decision rights delegation, and performance-The case of franchising. Journal of Small Business Management, v. 54, n. 3, p. 973-991, 2016.

HOUSE, R.; ROUSSEAU, D. M.; THOMASHUNT, M. The meso paradigma framework for the integration of micro and macro organizational-behavior. Research in Organizational Behavior, v. 17, p. 71-114, 1995.

ITTNER, C.D.; LARCKER, D.F. Innovations in performance measurement: trends and research implications. Journal of Management Accounting Research, v. 10, n. 1, p. 205, 1998.

JIANG, X.; JIANG, F.; CAI, X.; LIU, H. The mediating role of resource sharing. Industrial Marketing Management, v. 45, n. 1, p. 128-138, 2015.

JIANG, Z.; SHIU, E.; HENNEBERG, S.; NAUDE, P. Relationship quality in business to bussines relationships: Reviewing the current literatures and proposing a new measurement model. Psychology \& Marketing, v. 33, n. 4, p. 297-313, 2016.

KAPLAN, R.S.; NORTON, D.P. The balanced scorecard: measures that drive performance. Harvard Business Review, v. 70, n. 1, 71-79, 1992.
KASTNER, A. N.; MAHMOUND, M. A.; BUAME, S, C.; GABRAH, A. Y. Franchising in African markets: Motivations and challenges from a Sub-Saharan African country perspective. Thuderbird International Business Review, v. 61, n. 1, p. 169-181, 2019.

KWON, I. W. G.; SUH, T. Factors affecting the level of trust and commitment in supply chain relationships. Journal of Supply Chain Management, v. 40, n. 1, p. 4-14, 2004.

LEE, K. Knowledge sharing in franchise system: Franchisee self-leadership, satisfaction and compliance. International Journal of Contemporary Hospitality Management, v. 29, n. 12, p. 3101-3118, 2017.

MAHAMA, H. Management control systems, cooperation and performance in strategic supply relationships: A survey in the mines. Management Accounting Research, v. 17, n. 3, p. 315-339, 2006.

MAYER, R. C.; DAVIS. J. H.; SCHOORMAN, D. An integrative model of organizational trust. Academy of Management Review, v. 20, n. 1, p. 709-734, 1995.

MCAULAY, S. Non-contractual relations in business. American Sociological Review, v. 28, n. 1, p. 55-70, 1963.

MCKNIGHT, D. H.; CHOUDHURY, V.; KACMAR, C. Developing and validating trust measures for e-commerce: An integrative typology. Information Systems Research, v. 13, n. 3, p. 334-359, 2002.

MEAD, M. Cooperation and competition among primitive peoples. New York: Transaction Publishers, 1932.

MILES, R. E.; SNOW, C. C. Causes of failure in network organizations. California Management Review, v. 4, n. 1, p. 93-72, 1992. 
MOHR, J.; SPEKMAN, R. Characteristics of partnership success: partnership attributes, communication behavior, and conflict resolution techniques. Strategic Management Journal, v. 15, n. 2, p. 135-152, 1994.

MORGAN, R. M.; HUNT, S. D. The commitment-trust theory of relationship marketing. The Journal of Marketing, v. 58, n. 3, p. 20-38, 1994.

MOURITSEN, J.; THRANE, S. Accounting, network complementarities and the development of inter-organisational relations. Accounting, Organizations and Society, v. 31, n. 3, p. 241275, 2006.

PALMATIER, R. W.; DANT, R. P.; GREWAL, D. A comparative longitudinal analysis of theoretical perspectives of interorganizational relationship performance. Journal of Marketing, v. 71, n. 4, p. 172-194, 2007.

PARTANEN, J.; KOHTAMÄKI, M.; PATEL, P.C.; PARIDA, V. Supply chain ambidexterity and manufacturing SME performance: the moderating roles of network capability and strategic information flow. International Journal of Production Economics, v. 221, 107470, 2020.

RING, P. S.; VAN DE VEN, A. H. Developmental processes of cooperative interorganizational relationships. Academy of Management Review, v. 19, n. 1, p. 90-118, 1994.

ROUSSEAU, D. M.; SIRKIN, S. B.; BURT, R. S.; CAMERER, C. Not so different after all: A crossdiscipline view of trust. Academy of Management Review, v. 23, n. 1, p. 393-404, 1998.

SELNES, F; SALLIS, J. Promoting relationship learning. American Marketing Association, v. 67, n. 3, p. 80-95, 2003.
SMITH, K. G.; CARROLL, S. J.; ASHFORD, S. J. Intra-and interorganizational cooperation: Toward a research agenda. Academy of Management Journal, v. 38, n. 1, p. 7-23, 1995.

TIDSTROM, A.; HAGBERG-ANDERSSON, A. Critical events in time and space when cooperation turns into competition in business relationships. Industrial Marketing Management, v. 41, n. 2, p. 333-343, 2012.

TJOSVOLD, D. Cooperative and competitive goal approach to conflict: Accomplishments and challenges. Applied Psychology, v. 47, n. 3, p. 285-313, 1988.

TURNBULL, P.; OLIVER, N.; WILKINSON, B. Buyer-supplier relations in the UK-automotive industry: Strategic implications of the Japanese manufacturing model. Strategic Management Journal, v. 13, n. 2, p. 159-168, 1992.

VEREECKE, A.; MUYLLE, S. Performance improvement through supply chain collaboration in Europe. International Journal of Operations \& Production Management, v. 26, n. 11, p. 11761198, 2006.

WEBER, B.; HEIDENREICH, S. When and with whom to cooperate? Investigating effects of cooperation stage and type on innovation capabilities and success. Long Range Planning, v. 51, n. 2, p. 334-350, 2018.

ZUCKER, L. G. Production of trust: Institutional sources of economic structure. Research in Organizational Behavior, v. 8, n. 1, p. 18401920, 1986. 